\title{
Dinámica de inversión y competencia en generación eléctrica en un escenario de liberalización en Perú: la importancia de los contratos de largo plazo
}

\author{
Rivasplata R., ARNOLD \\ Pontificia Universidad Católica del Perú (Perú) \\ Correo electrónico: a.rivasplata.r@gmail.com \\ GARCÍA C., RAÚL \\ Pontificia Universidad Católica del Perú (Perú) \\ Correo electrónico: raul.garciac@pucp.pe
}

\begin{abstract}
RESUMEN
En este artículo se analiza el comportamiento de los grupos de generación eléctrica que toman decisiones sobre inversión y producción en un escenario de mercado liberalizado introduciendo contratos de largo plazo, en un contexto donde hay alto nivel de concentración de mercado. Para ello, se emplea un modelo determinístico dinámico de competencia imperfecta (con estrategias open loop) que se resuelve utilizando un sistema de ecuaciones complementarias mediante el software GAMS, a fin de proveer simulaciones que muestren los impactos de las diferentes estructuras de mercado (monopolio, oligopolio con franja competitiva, oligopolio (Cournot) y competencia perfecta) sobre las decisiones de inversión y producción, asumiendo la existencia de dos bloques horarios (base y pico) y considerando la existencia de dos tecnologías a utilizarse para el despacho eléctrico (hidráulica y térmica) con datos del año base 2011 para un horizonte temporal de 10 años. Los resultados muestran que a pesar de que en los diferentes escenarios de análisis puedan existir indicios de poder de mercado, existe un efecto positivo importante sobre la inversión en capacidad, la producción por tecnología, y precios por bloque de horario.
\end{abstract}

Palabras clave: decisiones de inversión, teoría de juegos, equilibrio de Nash, optimización, juegos dinámicos, poder de mercado, oligopolio, mercado eléctrico.

Clasificación JEL: C61; C73; L13; L94.

MSC2010: 47N10; 65K10; 91A25; 91A50; 91B24; 91B38.

Artículo recibido el 6 de enero de 2018 y aceptado el 27 de septiembre de 2018 


\title{
Investment dynamics and competition in electricity generation under liberalization scenario in Peru: the importance of long-term contracts
}

\begin{abstract}
We analyze the behavior of electricity generation groups that make investment and production decisions in a liberalized market scenario by introducing longterm contracts, in a context where there is a high level of market concentration. In this way, a dynamic deterministic model of imperfect competition (with open loop strategies) is used, which is solved using a system of complementary equations through GAMS software, in order to provide simulations that show the impacts of the different market structures (monopoly, oligopoly with competitive fringe, oligopoly (Cournot) and perfect competition) on the decisions of investment and production, assuming the existence of two segment loads (base and peak) and considering the existence of two technologies to be used for the electric power dispatch (hydraulic and thermal) with 2011 data for 10-year planning horizon. The results show us that although there may be indications of market power in the different analysis scenarios, there is a significant positive effect on investment in capacity, production by technology, and prices per segment loads.
\end{abstract}

Keywords: investment decisions, game theory, Nash equilibrium, optimization, dynamic games, market power, oligopoly, electricity market.

JEL classification: C61; C73; L13; L94.

MSC2010: 47N10; 65K10; 91A25; 91A50; 91B24; 91B38.

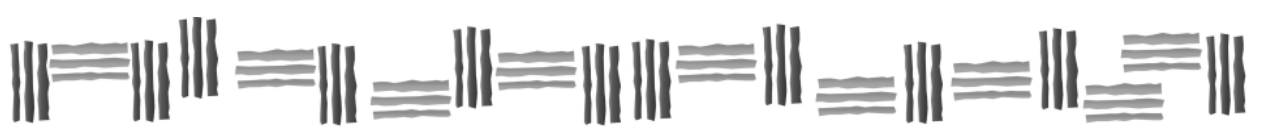




\section{Introducción.}

La industria eléctrica peruana, a partir de 1992, ha enfrentado un profundo proceso de reforma conducente a privilegiar la competencia y aumentar la eficiencia productiva en el sector en búsqueda de un parque generador óptimo que tenga incidencia sobre las tarifas de los usuarios finales. De esta manera, se dejó sin efecto la integración vertical y se estableció un régimen de libertad de precios para que los suministros puedan realizarse en condiciones de competencia y un sistema de precios regulados para aquellos suministros que por su naturaleza lo requieran (Ley de Concesiones Eléctricas-1992).

Con un mayor desarrollo del sector eléctrico al 2005, se presenció una situación de mejora en provisión de servicio y disminución de la incertidumbre, lo que fomentó una mayor participación del sector privado dado que, a diferencia de las empresas del Estado, no presentaba bajos niveles de productividad y tarifas por debajo de sus costos (Dammert, Gallardo \& García, 2005), generando un incremento de la oferta eléctrica con una mayor cobertura y una mejor calidad (servicio ininterrumpido).

Sin embargo, aspectos políticos (incertidumbre) y regulatorios propios del sector eléctrico, limitaron la entrada e inversión eficaz de empresas que podrían haber fomentado un entorno competitivo con efectos sobre el nivel tarifario. Es así que el mercado eléctrico no ha dejado de estar muy concentrado, con indicios de un ejercicio de poder de mercado ${ }^{1}$ que se genera, por ejemplo, con postergación de decisiones de inversión, desincentivo de conversión de centrales, indisponibilidad de centrales, retiros de capacidad, entre otros ${ }^{2}$. Producto de ello, del 2002 al 2011, el mercado eléctrico solo pasó de ser altamente concentrado a moderadamente concentrado; es decir, el índice de concentración Herfindal-Hirschman tuvo una reducción de 3001 a 2072. Para eso, se contaron con grupos económicos que participan simultáneamente en diversas tecnologías (hidráulicas y térmicas), entre las cuales se encuentran el Estado, Endesa, Duke Energy, SN Power, Suez, Globoleq, Otros, de los cuales los dos primeros son dominantes en función a su participación, y también comparado con los que recién entran a operar que cuentan con una baja capacidad de generación.

El problema identificado es que la concentración del mercado no ha generado una presión para fomentar una eficiencia productiva y una adecuación más eficiente del parque generador, a través de las decisiones de inversión. Esto se basa en la estrecha relación entre la concentración de mercado con el ejercicio de poder de mercado, que ha limitado los incentivos de inversión en capacidad en tecnologías más eficientes, de tal manera que se tenga un parque generador que no está bien diversificado en tecnologías más económicas que pueden proveer igual o mejor a las ya presentes, y que a su vez exista un efecto positivo sobre las tarifas.

Sobre las decisiones de inversión y sus extensiones existe una amplia literatura, desde el enfoque estático hasta el dinámico, enfatizando para el sector eléctrico los estudios que exploran la estructura de mercado. Un primer análisis estático es desarrollado por Arellano y Serra (2005) que demostraron como el ejercicio de poder de mercado distorsiona las decisiones de inversión. Un análisis dinámico para analizar las estrategias de inversión de los competidores o generadores se muestra en Pineau y Murto (2003), Genc et al. (2007), Burger y Ferstl (2008), Genc y Thille (2011), Zoettl (2008). Para el sector eléctrico peruano, De la Cruz y García (2002) analizaron las conductas estratégicas de las generadoras asumiendo una declaración de costos para el despacho; Dammert et al. (2005) analizaron la inversión en la actividad de generación considerando la problemática de poder de mercado; Gallardo et al. (2005a) y Gallardo et al. (2005b) mostraron aspectos que pueden afectar la inversión en la actividad de generación.

\footnotetext{
${ }^{1}$ Las empresas pueden ejercer poder de mercado cuando alteran el portafolio de generación que no es óptimo (Arellano y Serra, 2005).

${ }^{2}$ La libertad de inversión en capacidad, genera que las empresas tengan estrategias sobre sus decisiones considerando a las de otros (existentes o potenciales entrantes) en el mercado (González, 2008).
} 
Tomando en cuenta que la decisión en los procesos de inversión en la actividad muestra que si bien las empresas manipulaban la regla de precios (tarifa de barra) hasta el 2006 mediante la disminución de invertir a futuro o por indisponibilidad, también se aprovechaba la rentabilidad de las centrales de diésel, visto que representaba un costo hundido y por la falta de competencia en el sector porque representaba un costo variable alto ejerciendo una presión al alza de los precios. Posteriormente, con el mecanismo de las licitaciones se buscó reducir el ejercicio de poder de mercado de los generadores e incentivarlos a contar con nuevas centrales de generación para poder abastecer la demanda creciente de los distribuidores, producto del aumento del consumo de electricidad.

El objetivo de este trabajo es analizar la importancia de los contratos de largo plazo y de la franja competitiva sobre las decisiones de inversión en capacidad, producción, precios de generación bajo un análisis dinámico de oligopolio para un horizonte temporal de 10 años. Para ello, se busca mostrar cómo interactúan los grupos económicos de generación que compiten a lo Cournot bajo el enfoque de teoría de juegos siguiendo a Pineau et al. (2011) y Pineau y Murto (2003), considerando que estos no están regulados en su decisión de producción, y no existen tarifas en barra definidas por el ente regulador.

En esa línea, es importante responder algunas preguntas que se derivan de la problemática planteada: ¿Cuál es el efecto de los contratos de largo plazo bajo un escenario de mercado liberalizado, sobre la inversión en capacidad, la producción y el precio? ¿Cuál sería la composición del parque generador con tecnologías hidráulica y térmica? ¿Qué pasa con las variables de elección cuando el mercado está altamente concentrado y se toma en cuenta el tamaño franja competitiva? ¿Cuál es la relevancia de dicho escenario para las decisiones estratégicas de los competidores?

El presente trabajo se desarrolla en 4 secciones. En la sección 2 se desarrolla el modelo dinámico de oligopolio y metodología. En la sección 3 se reportan los datos a utilizar en la simulación del modelo y se discuten los resultados para los casos de competencia perfecta, oligopolio (Cournot), oligopolio con franja competitiva y monopolio bajo escenarios sin contratos de largo plazo y con estos. Finalmente, en la sección 4, se desarrollan las conclusiones y recomendaciones.

\section{Metodología y modelo.}

La competencia en el sector eléctrico peruano y las conductas estratégicas de los grupos económicos de generación eléctrica, que cuentan solo con disponibilidad para la toma de decisiones de inversión y con un alto nivel de concentración, permiten desarrollar un análisis dinámico de inversión basado en el enfoque de teoría de juegos. Las fusiones horizontales, permiten a un grupo operar con distintas tecnologías y abastecer diferentes bloques de horario (punta y fuera de punta). Asimismo, la actividad de generación se plantea bajo un análisis de mercado oligopólico, y la competencia de los grupos de generación, por las características del sector, se desarrolla como un modelo Cournot.

Por lo descrito, se considera un mecanismo de solución que se desarrolla mediante una calibración en GAMS (General Algebraic Modeling System), obteniendo los equilibrios de un modelo de oligopolio dinámico desarrollado como un MCP (Mixed Complementary Problem). Para esto se toma en cuenta a todos los grupos con datos iniciales de 2011, y se plantean cuatro escenarios para analizar el cambio en las variables de elección. El primero asume que todos los grupos son tomadores de precios (competencia perfecta), el segundo considera que solo existe un gran grupo generador (monopolio), el tercero asume que todos los grupos deciden estratégicamente (oligopolio), y finalmente cuando existe una franja competitiva donde los pequeños grupos compiten. 
Generalizando el problema de maximización a implementar, una empresa tiene como objetivo obtener mayores beneficios de la siguiente manera:

$$
\begin{gathered}
\max \pi=I T-C T \\
\max \pi=p Q_{t}-C\left(Q_{t}, K_{t}\right)-\Psi\left(I_{t}\right) \\
\text { s.a } \\
\begin{array}{lr}
\text { (1) capacidad del sistema } & K_{t+1}=(1-\delta) K_{t}+I_{t} \\
\text { (2) capacidad de producción } & Q_{t} \leq h K_{t} \\
\text { (3) no negatividad } & Q_{t}, I_{t} \geq 0
\end{array}
\end{gathered}
$$

donde $p$ es el precio de electricidad, $Q_{t}$ es la energía producida, $C\left(Q_{t}, K_{t}\right)$ es costo de producción, $\Psi\left(I_{t}\right)$ es la función de inversión, $K_{t}$ es la capacidad de generación, $I_{t}$ es inversión en capacidad de generación, y $h$ es el número de horas. El problema a maximizar se modifica de la siguiente manera:

$$
\max \pi_{i}=\bar{Q}_{c i} \cdot \bar{p}_{c i}+\left[Q_{i}-Q_{c i}\right] \cdot p_{s}(Q)-C\left(Q_{t}, K_{t}\right)-\Psi\left(I_{t}\right)
$$

donde $\bar{Q}_{c i}$ es la cantidad contratada que se concreta al distribuidor al precio $\bar{p}_{c i}$ que está fijo por los años que dure el contrato. Al mercado spot se llega a vender la diferencia.

El modelo a seguir muestra que existe un número limitado de grupos eléctricos (jugadores), $\mathrm{N}=\{1, \ldots, \mathrm{n}\}$ que compiten en el mercado. Las tecnologías de generación asumidas son las hidroeléctricas y térmicas, que abastecen a demandas base y pico. Para esto se toma como referencia lo propuesto por Dammert et al. (2005), Pineau y Murto (2003) y Pineau et al. (2011). La generalización del modelo se expresa:

$$
\begin{gathered}
\max _{j}=\sum_{t=0}^{T} \beta^{t}\left\{p^{h p}\left(Q_{t}^{h p}, e_{t}\right) Q_{t}^{h p}+p^{h f p}\left(Q_{t}^{h f p}, e_{t}\right) Q_{t}^{h f p}-C^{H}\left(q^{H, h p}+q^{H, h f p}, K_{t}^{H}\right)-C^{T}\left(q^{T, h p}+q^{T, h f p}, K_{t}^{T}\right)\right. \\
\left.-\Psi^{H}\left(I_{t}^{H}\right)-\Psi^{T}\left(I_{t}^{T}\right)\right\}
\end{gathered}
$$

Considerando que la demanda eléctrica en el Perú está sujeta a mucha variabilidad en hora punta, no se realiza un análisis sobre la curva de carga para el modelo, por lo que se asume una demanda por bloque tipo lineal ${ }^{3}$ y decreciente que caracteriza la cantidad total despachada en el mercado:

$$
\begin{aligned}
p^{h p} & =A^{h p}-G Q \\
p^{h f p} & =A^{h f p}-G Q
\end{aligned}
$$

En un escenario donde el mercado no cuenta con contratos, los grupos van a buscar maximizar sus beneficios teniendo como variables de elección la cantidad producida y la inversión. El problema de optimización está representado por:

$$
\begin{gathered}
\max \pi_{j}=\sum_{t=0}^{9} \beta^{t}\left\{p^{h p}\left(Q_{t}^{h p}\right)\left[Q_{j}\right]_{t}^{h p}+p^{h f p}\left(Q_{t}^{h f p}\right)\left[Q_{j}\right]_{t}^{h f p}-C^{H}\left(q^{H, h p}+q^{H, h f p}, K_{t}^{H}\right)-C^{T}\left(q^{T, h p}+q^{T, h f p}, K_{t}^{T}\right)\right. \\
\left.-\Psi^{H}\left(I_{t}^{H}\right)-\Psi^{T}\left(I_{t}^{T}\right)\right\}
\end{gathered}
$$

En un contexto de oligopolio donde los grupos eléctricos operan en todas las tecnologías, y en el que se introducen contratos de largo plazo, el nuevo problema de optimización ${ }^{4}$ es representado por:

\footnotetext{
${ }^{3}$ Siguiendo a Dammert et al. (2005) y Pineau et al. (2011) se asume que el parámetro $A$ crece a una tasa g, representando esta la tasa de crecimiento de la demanda $A^{t}=(1+g)^{t} A$, de los dos bloques horarios.

${ }^{4}$ Se impone la restricción de cantidad contratada bajo el precio pactado. La producción total de energía se complementa con las diferencias de la cantidad total menos la contratada por los precios por bloque de horario.
} 


$$
\begin{gathered}
\max \pi_{j}=\sum_{t=0}^{9} \beta^{t}\left\{\bar{Q}_{c j}^{h p} * \bar{p}_{c j}^{h p}+\bar{Q}_{c j}^{h f p} * \bar{p}_{c j}^{h f p}+p^{h p}\left(Q_{t}^{h p}\right)\left[Q_{j}-Q_{c j}\right]_{t}^{h p}+p^{h f p}\left(Q_{t}^{h f p}\right)\left[Q_{j}-Q_{c j}\right]_{t}^{h f p}\right. \\
q, I \\
\left.\left.C^{H, h p}+q^{H, h f p}, K_{t}^{H}\right)-C^{T}\left(q^{T, h p}+q^{T, h f p}, K_{t}^{T}\right)-\Psi^{H}\left(I_{t}^{H}\right)-\Psi^{T}\left(I_{t}^{T}\right)\right\} \\
\text { s.a: } \\
K_{t+1}^{i}=(1-\delta) K_{t}^{i}+I_{t}^{i} \quad i=H, T \\
q_{t}^{i, h p} \leq h p . K_{t}^{i} \\
q_{t}^{i, h f p} \leq h f p . K_{t}^{i} \\
q_{t}^{i, h p}, q_{t}^{i, h f p}, I_{t}^{i} \geq 0
\end{gathered}
$$

donde:

$\bar{Q}_{c j} \quad$ Energía contratada $(M W h)$

$Q_{j} \quad$ Energía total producida (vendida) por empresa $j$

$Q \quad$ Energia total producida (vendida) en el mercado

$q^{H, h p}, q^{H, h f p}$ Energía hidraúlica en diferentes bloques de horario $(M W h)$

$q^{T, h p}, q^{T, h f p}$ Energía térmica en diferentes bloques de horario (MWh)

$I_{t}^{H}, I_{t}^{T} \quad$ Inversión en capacidad de generación $(M W)$

$K_{t}^{H}, K_{t}^{T} \quad$ Capacidad de generación $(M W)$

$\bar{p}_{c j} \quad$ Precio pactado en contrato bilateral (mercado regulado)

$\beta \quad$ Tasa de descuento intertemporal $0<\beta<1$

$\delta \quad$ Tasa de depreciación

La capacidad instalada inicial por tipo de tecnología para cada grupo de generación eléctrica está representado por $K_{0}^{i}$, mientras que la inversión en capacidad inicial se deriva de los costos de inversión $I_{0}^{i}$.

Los costos de producción se caracterizan siguiendo lo propuesto por Pineau y Murto (2003), de tal manera que para las centrales hidráulicas, el costo marginal depende positivamente de la producción, mientras que para las centrales térmicas, el costo marginal depende de la capacidad y de la producción. Los costos de producción para tecnologías hidráulica y térmica:

$$
\begin{gathered}
C^{H}\left(q^{H}, K_{t}^{H}\right) \triangleq C^{H}\left(q^{H}\right)=\alpha_{H} \cdot q^{H} \\
C^{T}\left(q^{T}, K_{t}^{T}\right)=\alpha_{T} \cdot q^{T}+\frac{\gamma K_{t}}{\phi+1}\left(\frac{q^{T}}{K_{t}}\right)^{\phi+1}
\end{gathered}
$$

Se incorporan costos de inversión cuadrática dado que representan, por un lado, una función de centrales térmicas que a su vez disponen de más de una tecnología, lo que permite caracterizar mejor los costos de centrales por su capacidad (ciclo simple y/o ciclo combinado). Para ello, se asume que esta función es convexa y creciente en inversión en capacidad. Asimismo, para las centrales hidráulicas, se considera una función que depende de la capacidad e inversión en capacidad, de tal manera que la inversión marginal incrementa con la capacidad instalada. De esta manera los costos de inversión para tecnologías hidráulica y térmica:

$$
\begin{gathered}
\Psi\left(I_{t}^{H}\right)=\psi_{1} I_{t}+\frac{1}{2} \psi_{2} I_{t}^{2}, \text { donde } \psi_{1}=\kappa K_{t} \\
\Psi\left(I_{t}^{T}\right)=\frac{1}{2} \varphi I_{t}^{2}
\end{gathered}
$$

Asimismo, las decisiones de cada empresa sobre la cantidad a producir y la inversión en generación se expresan en el siguiente vector:

$$
x_{i}=\left[\left(q_{i m}^{t}, \forall m \in\{h p, h f p\}, t=0, \ldots, T\right) ;\left(I_{i m}^{t}, \forall m \in\{h p, h f p\}, t=0, \ldots, T-1\right)\right]
$$


El conjunto de acciones de cada jugador o empresa $i$ es $\Omega_{i}$; mientras que las acciones de todos los jugadores es determinado por $\Omega=\prod_{i \in N} \Omega_{i}$, que para el presente estudio $N$ representa 7 jugadores. En el modelo se utiliza la estructura de información "open loop" donde los jugadores basan sus decisiones en el tiempo y no son acumulativas en cada etapa. Entonces, el vector $x^{*}=$ $\left\{x_{1}^{*}, \ldots, x_{N}^{*}\right\} \in \Omega$ va a ser el equilibrio de Nash $^{6}$ si:

$$
\begin{aligned}
\pi_{i}\left(x^{*}\right) \geq & \pi_{i}\left(x_{1}^{*}, \ldots, x_{i-1}^{*}, x_{i}, x_{i+1}^{*}, \ldots, x_{n}^{*}\right), \forall x_{i} \in \Omega_{i}, \forall i \in N \\
& -\left\langle\nabla_{x_{i}} \pi_{i}\left(x^{*}\right), x_{i}-x_{i}^{*}\right\rangle \geq 0, \quad \forall x_{i} \in \Omega_{i}
\end{aligned}
$$

Se desarrolla el Lagrangiano con la restricción de la ecuación de acumulación del capital (variable rezagada) y la restricción de producción:

$$
\begin{aligned}
& L_{j}^{t}=\bar{Q}_{c j} \cdot \bar{p}_{c j}+p^{h p}\left(Q_{t}^{h p}\right)\left[Q_{j}-Q_{c j}\right]_{t}^{h p}+p^{h f p}\left(Q_{t}^{h f p}\right)\left[Q_{j}-Q_{c j}\right]_{t}^{h f p}-C^{H}\left(q^{H, h p}+q^{H, h f p}, K_{t}^{H}\right) \\
& -C^{T}\left(q^{T, h p}+q^{T, h f p}, K_{t}^{T}\right)-\Psi^{H}\left(I_{t}^{H}\right)-\Psi^{T}\left(I_{t}^{T}\right)+\sum_{i \in\{H, T\}} \lambda_{t+1}^{i}\left(I_{t}^{i}-\delta K_{t}^{i}\right)+\sum_{i \in\{H, T\}} \xi_{t}^{i}\left(h \cdot K_{t}^{i}-q_{t}^{i, h}\right)
\end{aligned}
$$

Resolviendo las condiciones de primer orden:

$$
\begin{gathered}
\frac{\partial L_{j}^{t}}{\partial q_{j}^{h f p}}=A^{h f p}-B^{h f p} Q_{h f p}-C^{h f p} Q_{h p}-B_{h f p}\left[Q_{j}-Q_{c j}\right]^{h f p}-C_{h p}\left[Q_{j}-Q_{c j}\right]^{h f p}-\alpha_{H}-\xi^{H} \leq 0, \\
j \in N \\
\frac{\partial L_{j}^{t}}{\partial q_{j}^{h p}}=A^{h p}-B^{h p} Q_{h p}-C^{h p} Q_{h f p}-C_{h f p}\left[Q_{j}-Q_{c j}\right]^{h p}-B_{h p}\left[Q_{j}-Q_{c j}\right]^{h p}-\alpha_{T}-\gamma\left(\frac{q^{T}}{K_{t}}\right)^{\phi}-\xi^{T} \leq 0, \\
j \in N \quad j \in N \\
\frac{\partial L_{j}^{t}}{\partial l_{j}^{h f p=H}}=-\left(\psi_{1}+\psi_{2} I\right)+\lambda_{t+1}^{H} \leq 0, \quad j \in N \\
\frac{\partial L_{j}^{t}}{\partial I_{j}^{h p=T}}=-\varphi I+\lambda_{t+1}^{T} \leq 0, \quad j \in N \\
\frac{\partial L_{j}^{t}}{\partial \xi_{j}^{h f p} p_{H}}=h f p . K_{t}^{i}-q_{t}^{i, h f p} \geq 0, \quad j \in{ }^{t} \\
\frac{\partial L_{j}^{t}}{\partial \xi_{j}^{h p=T}}=h p . K_{t}^{i}-q_{t}^{i, h p} \geq 0, \quad j \in N
\end{gathered}
$$

Representación de variables de coestado:

$$
\begin{gathered}
\lambda_{t+1}^{H}-\frac{\lambda_{t}^{H}}{\beta}=\kappa I_{t}+\delta \lambda_{t+1}^{H}-\xi_{t}^{H} h f p, \quad t \in \mathrm{T} \\
\lambda_{t+1}^{T}-\frac{\lambda_{t}^{T}}{\beta}=-\phi \frac{\gamma}{\phi+1}\left(\frac{q_{t}^{T}}{K_{t}^{T}}\right)^{\phi+1}+\delta \lambda_{t+1}^{T}-\xi_{t}^{T} h p, \quad t \in \mathrm{T}
\end{gathered}
$$

\footnotetext{
${ }^{5}$ Rasata y Zaccour (2010) reportan que una estructura de información open loop supone que el horizonte es finito, mientras que las inversiones en close loop supone un juego infinito.

${ }^{6}$ Nagurney (2002) hace referencia sobre el teorema de desigualdad variacional que está relacionado con el equilibrio derivado. Para esto se considera un supuesto de que el vector $x^{*}=\left\{x_{1}^{*}, \ldots, x_{n}^{*}\right\}$ es un equilibrio si y solo sí $x^{*} \in \Omega$ es una solución del método descrito. Entonces:
}

$$
\begin{gathered}
\left\langle F\left(x^{*}\right), x-x^{*}\right\rangle \geq 0, \quad \forall x \in \Omega \\
F(x) \equiv\left(-\nabla_{x_{1}} \pi_{1}(x), \ldots,-\nabla_{x_{n}} \pi_{n}(x)\right), \text { donde } \\
\nabla_{x_{i}} \pi_{i}(x)=\left(\frac{\partial \pi_{i}(x)}{\partial x_{1}}, \ldots, \frac{\partial \pi_{i}(x)}{\partial x_{n}}\right)
\end{gathered}
$$


Representación de las ecuaciones de estado:

$$
\begin{aligned}
K_{t+1}^{H} & =(1-\delta) K_{t}^{H}+I_{t}^{H i}, \quad t \in \mathrm{T} \\
K_{t+1}^{T} & =(1-\delta) K_{t}^{T}+I_{t}^{T}, \quad t \in \mathrm{T}
\end{aligned}
$$

Complementariamente, se precisan algunas limitaciones del modelo previamente planteado. No se incorpora la utilización de tecnologías renovables no convencionales (eólica, solar, hidráulica con capacidad menor a 20MW) en el parque generador, ni tampoco el efecto del subsidio que se le paga a estas tecnologías mediante el D.L $1002^{7}$, ya que se requeriría un tratamiento especial mediante algún componente estocástico, y que no cuenta con mucha representatividad en el consumo nacional de energía (menos del 5\%), lo que no reflejaría una distorsión significativa en los resultados que se presentan en el siguiente capítulo.

Otro aspecto que no se considera dentro del modelo planteado son las restricciones de transmisión eléctrica que afectan la configuración del parque generador, de tal manera que la congestión de las líneas no son incorporados en las decisiones de los agentes generadores al momento de decidir invertir en nueva capacidad y generar electricidad, dado que esto podría incentivar a los generadores a ejercer poder de mercado.

\section{Resultados: datos y simulación.}

En la industria eléctrica se han identificado 7 grupos de generación eléctrica que operan con tecnologías térmicas e hidráulicas, de los cuales, el Estado y Endesa son los más importantes por su participación en el mercado y el presente trabajo se enfoca en estos como los competidores en un contexto de mercado eléctrico liberalizado donde se define precio, cantidad, e inversión. En la tabla 1 se muestra la capacidad instalada y producción.

\begin{tabular}{|c|c|c|c|}
\hline Grupo Económico & Tecnología & Capacidad instalada (MW) & Producción total (GWh) \\
\hline \multirow{2}{*}{ Estado } & hidráulico & 1422 & \multirow{2}{*}{10249} \\
\hline & térmico & 296 & \\
\hline \multirow{2}{*}{ Endesa } & hidráulico & 753 & \multirow{2}{*}{10009} \\
\hline & térmico & 1174 & \\
\hline \multirow{2}{*}{ Duke Energy } & hidráulico & 351 & \multirow{2}{*}{2806} \\
\hline & térmico & 547 & \\
\hline \multirow{2}{*}{ SN Power } & hidráulico & 264 & \multirow{2}{*}{1685} \\
\hline & térmico & - & \\
\hline \multirow{2}{*}{ Suez } & hidráulico & 130 & \multirow{2}{*}{4675} \\
\hline & térmico & 956 & \\
\hline \multirow{2}{*}{ Globoleq } & hidráulico & - & \multirow{2}{*}{3994} \\
\hline & térmico & 602 & \\
\hline \multirow{2}{*}{ Otros } & hidráulico & 313,6 & \multirow{2}{*}{1799} \\
\hline & térmico & 177 & \\
\hline
\end{tabular}

Tabla 1. Capacidad en el mercado eléctrico peruano, 2011.

Fuente: Elaboración propia a partir de MINEM - Documento Promotor 2012, OSINERGMIN - Boletín Anual 2011.

\footnotetext{
${ }^{7}$ Decreto Legislativo 1002 (Decreto Legislativo de promoción de la inversión para la generación de electricidad con el uso de energías renovables).
} 
La calibración del modelo propuesto permite analizar un posible escenario en contraste con la verdadera situación del sector eléctrico. Para esto, se consideran parámetros como los propuestos en Dammert et al. (2005) y Pineau et al. (2011). Asimismo, se considera que el periodo de operación de las centrales eléctricas es anual (365 días x 24 horas $=8760$ horas), y asumiendo que por lo general las centrales térmicas operan en hora punta que representan en promedio 4 horas diarias y la incorporación del gas natural (ciclo simple y ciclo combinado), se tiene dos bloques de horario representados por base (5760 horas) y pico (3000 horas).

Por otro lado, se consideran costos referenciales por tipo de tecnología que abastecen a los bloques de horario. Por ejemplo, en la tabla 2 se observa la relación negativa entre el costo de inversión (MW-año) y el costo variable (MWh), dado que implementar una central hidráulica es más costoso que una térmica.

Tabla 2. Costos estándares de energía eléctrica (miles de US\$).

\begin{tabular}{|c|c|c|c|}
\hline Central & Inversión (MW) & Costo fijo anual (MW - Año) & Costo variable (MWh) \\
\hline Diésel & 350 & 55,12 & 74,4 \\
\hline Gas natural - CS & 400 & 63 & 18,2 \\
\hline Gas natural - CC & 550 & 86,62 & 11,9 \\
\hline Hidráulica & 1250 & 176,63 & 0,9 \\
\hline
\end{tabular}

Fuente: Dammert et al. (2010).

Los precios para cada demanda que abastecen las empresas generadoras se determinan en base a la agregación del precio de potencia y de energía obtenidos de los valores referenciales de los puntos de conexión del Sistema Eléctrico Interconectado Nacional. El precio contratado ${ }^{8}$ por bloque de horario se basa en los factores de actualización al 2011 de precios a nivel de generación adjudicados. Para considerar la cantidad que cada grupo contrata con las distribuidoras en el mercado eléctrico se recurre al Balance de Generación Eléctrica por Interconexión y Empresas de Osinergmin - GART, donde se encuentran datos sobre la venta de energía por empresa. También, los parámetros para la simulación se obtienen de datos estándares para un mercado eléctrico mostrados por Dammert et al. (2005), Dammert et al. (2010), entre otros.

Los resultados del análisis de la actividad de generación eléctrica, consideran cuatro escenarios de competencia de mercado para cada contexto. Estos permiten comparar las respuestas de las estrategias de los diferentes grupos de generación y analizar la importancia de los contratos de largo plazo cuando se liberaliza el mercado.

La evolución del nivel de precios en bloque de horario (gráfico 1) base muestra un comportamiento decreciente durante los seis primeros años, a excepción de una situación en la que hay un solo generador que abastece al sector. El efecto de los contratos muestra una reducción de 24,48\% para el monopolio, 9,73\% para el oligopolio (Cournot), 9,83\% para el oligopolio con franja competitiva. Para el monopolio se puede notar que los precios son mayores por el poder de mercado y la ausencia de potenciales grupos que no muestran incentivos a entrar; mientras que, en competencia perfecta, el resultado muestra el mismo comportamiento con la incorporación de contratos, pero la evolución de los precios tiende a ser más pronunciada. Asimismo, la franja competitiva permite que exista una demanda residual abastecida bajo precios ya determinados en el mercado y que haya una disminución del poder de mercado. Este efecto en los precios es mayor ante una situación de oligopolio (Cournot), pero el nivel de precios se reduce más para el oligopolio con franja competitiva.

\footnotetext{
${ }^{8}$ Resolución OSINERGMIN Nº193-2011-OS/CD.
} 
Gráfico 1. Evolución del precio base (\$/MWh).

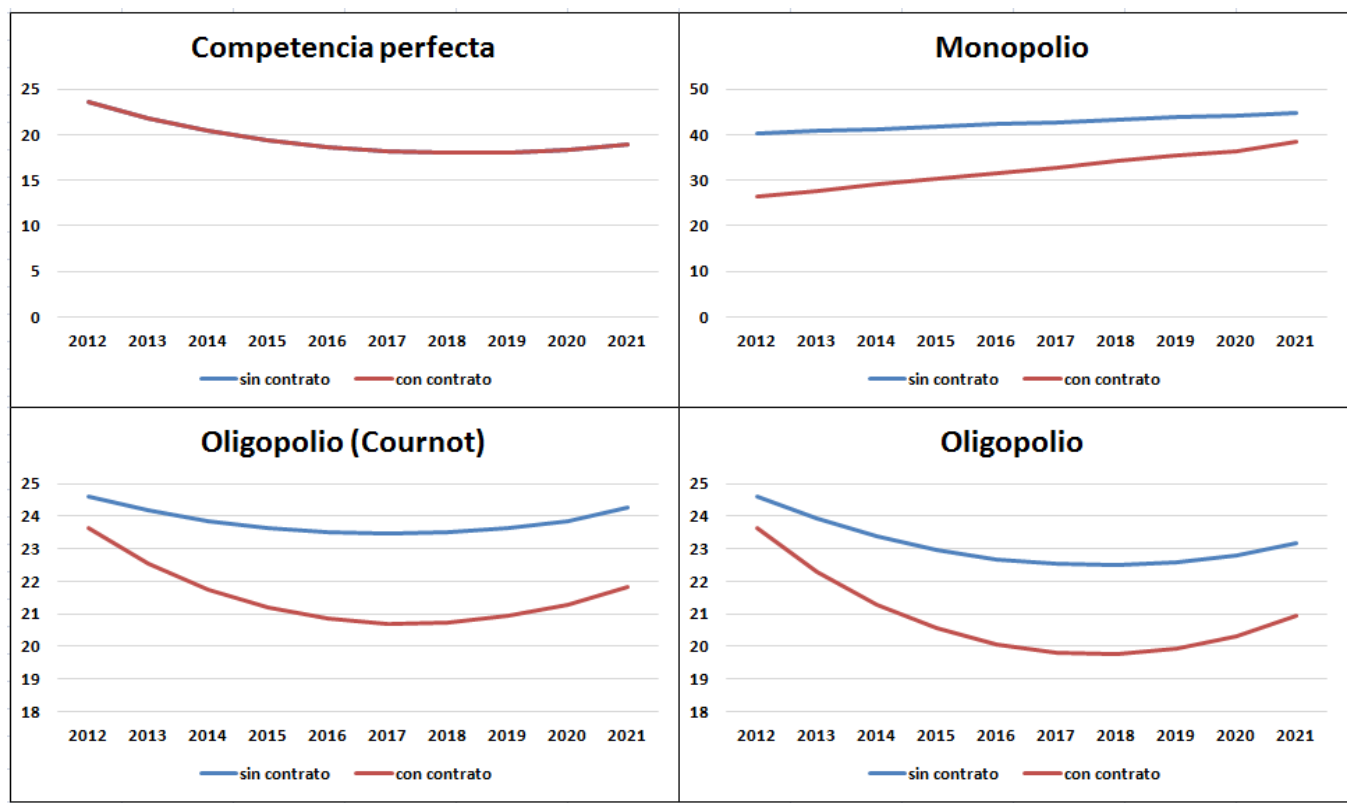

Fuente: Elaboración propia.

El precio en el bloque horario pico (gráfico 2) muestra una clara diferencia con el resultado previo dado que se opera con tecnologías de combustión que implican costos marginales mayores ante la presión de una mayor demanda concentrada en un margen de horas. A excepción de competencia perfecta, se observa un crecimiento constante para todos los escenarios. El efecto de los contratos muestra una reducción de 16,32\% para el monopolio, 7,62\% para el oligopolio (Cournot), 4,45\% para el oligopolio con franja competitiva. En competencia perfecta, la situación permanece inalterada. Asimismo, el efecto sobre el mercado que cuente con oligopolio (Cournot) es mayor a una situación de franja competitiva. Sin embargo, el nivel de precios del escenario de este último, presenta una mayor reducción a partir del cuarto año.

Gráfico 2. Evolución del precio pico (\$/MWh).

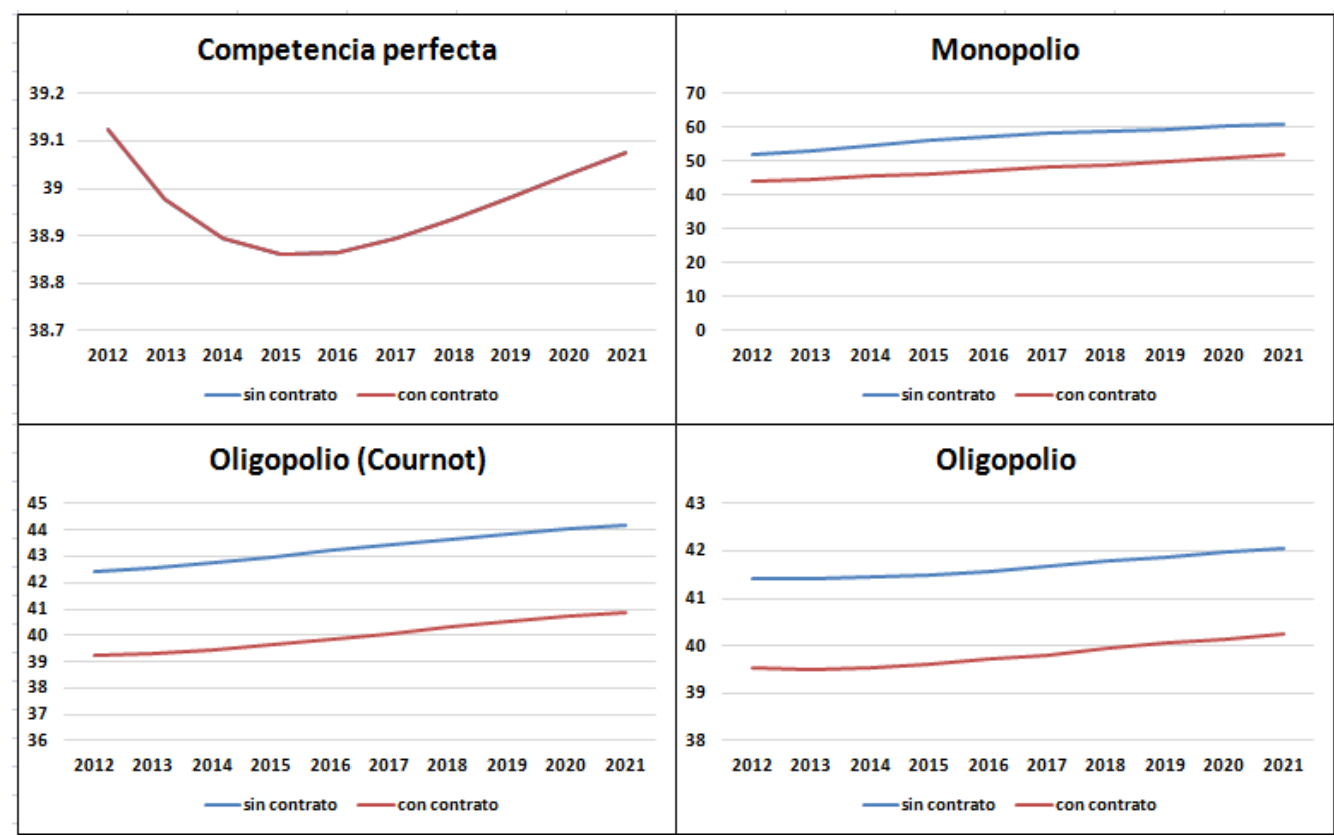

Fuente: Elaboración propia. 
Si se toman en cuenta los contratos, la situación referida a los precios mejora porque disminuiría el poder de mercado que se pueda ejercer bajo la liberalización. La baja competencia entre los grupos permite que se venda energía a precios altos, pero cuando se contrata por varios años, restringiendo la influencia sobre el precio, la cantidad de energía que queda en el mercado spot se podría vender a un precio menor.

En cuanto a la inversión en capacidad de tecnología hidráulica (gráfico 3), el efecto de los contratos muestra un incremento de $123,62 \%$ para el monopolio, 12,15\% para el oligopolio (Cournot), 7,18\% para el oligopolio con franja competitiva. Se muestra que bajo en un escenario de competencia perfecta, se tiene un incentivo a contar con mayor capacidad a corto plazo; también se observa que una incorporación de contratos no altera la evolución del escenario inicial. Otro escenario particular es el monopolio, donde el único grupo invierte crecientemente, pero con un nivel bajo, lo que se justificaría el poco incentivo a contar con centrales hidráulicas o invertir en mayor capacidad, lo que muestra que un costo fijo alto y costo variable bajo le genera poca rentabilidad al grupo monopolista. La inversión mejora con contratos, pero a partir de la segunda mitad, hay una desaceleración, reflejando un incentivo parcial. Analizando los casos que se ajustan al sector, el oligopolio con franja competitiva genera una inversión inicial mayor en capacidad que el resto, y presenta una reducción gradual durante el periodo de análisis. La incorporación de contratos en el modelo permite un incremento de la inversión.

Gráfico 3. Inversión en tecnología hidráulica (MW).

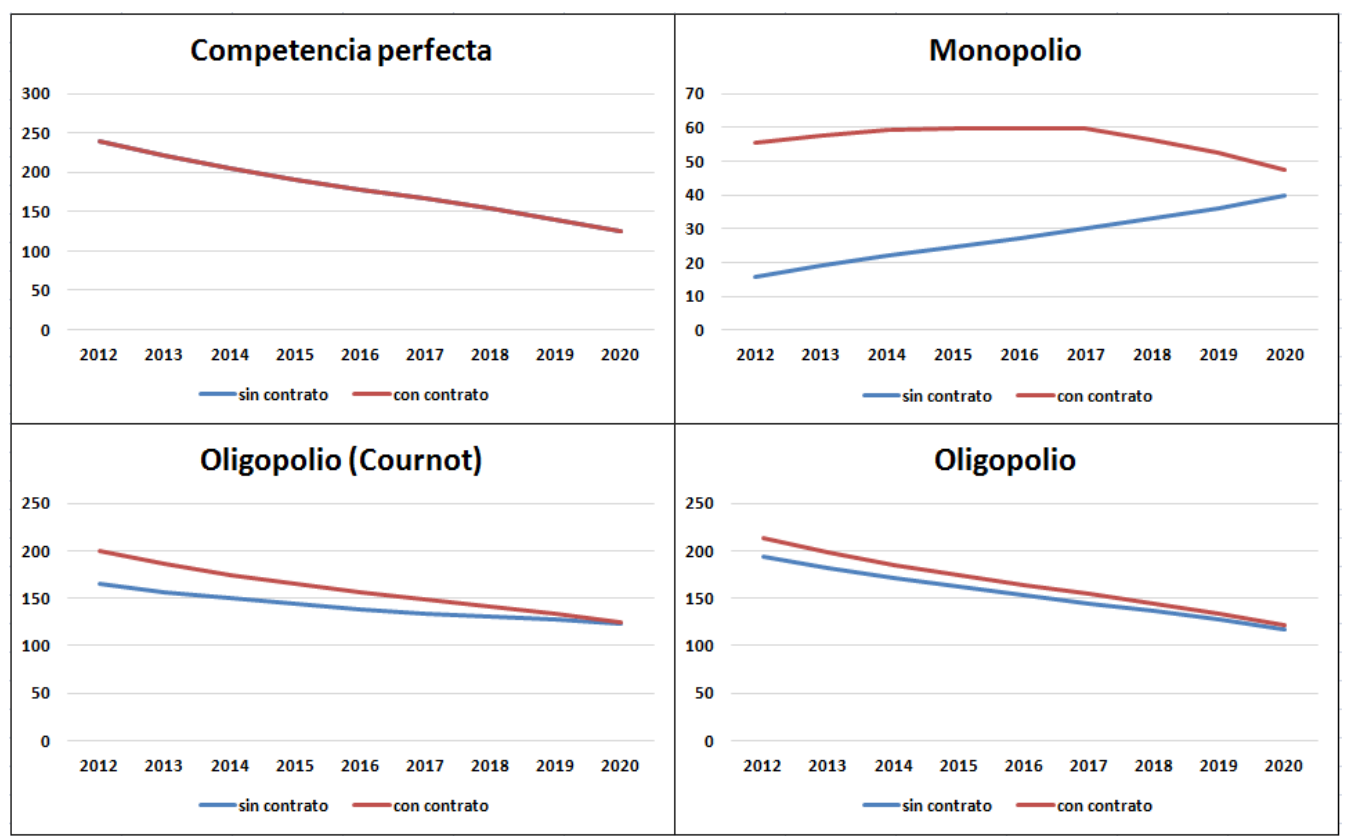

Fuente: Elaboración propia.

La tecnología térmica por su nivel de operatividad y rentabilidad, presenta una velocidad de crecimiento de inversión mayor a la tecnología hidráulica, en todos los escenarios (gráfico 4). El efecto de los contratos muestra un incremento de 15,22\% para el monopolio, 1,37\% para el oligopolio (Cournot) y una reducción del 2,27\% para el oligopolio con franja competitiva. Bajo competencia perfecta, los grupos del sector son tomadores de precios por lo que cuentan con incentivos para invertir en centrales o capacidad de manera creciente. Para el caso de monopolio se observa un menor crecimiento, lo que podría hace suponer que invierte solo en capacidad de centrales ya operativas. Situación similar a la de competencia perfecta se ve reflejada en los escenarios de oligopolio con franja competitiva, y de oligopolio (Cournot), siendo el primero en tener un ligero incentivo en mayor capacidad. En el contexto de contratos, el incentivo a invertir en la tecnología hidráulica es mayor a corto plazo que las térmicas, incluso se observa para el 
caso de oligopolio con franja competitiva un incentivo a subinvertir en tecnología térmica y sobreinvertir en tecnología hidráulica.

Gráfico 4. Inversión en tecnología térmica (MW).

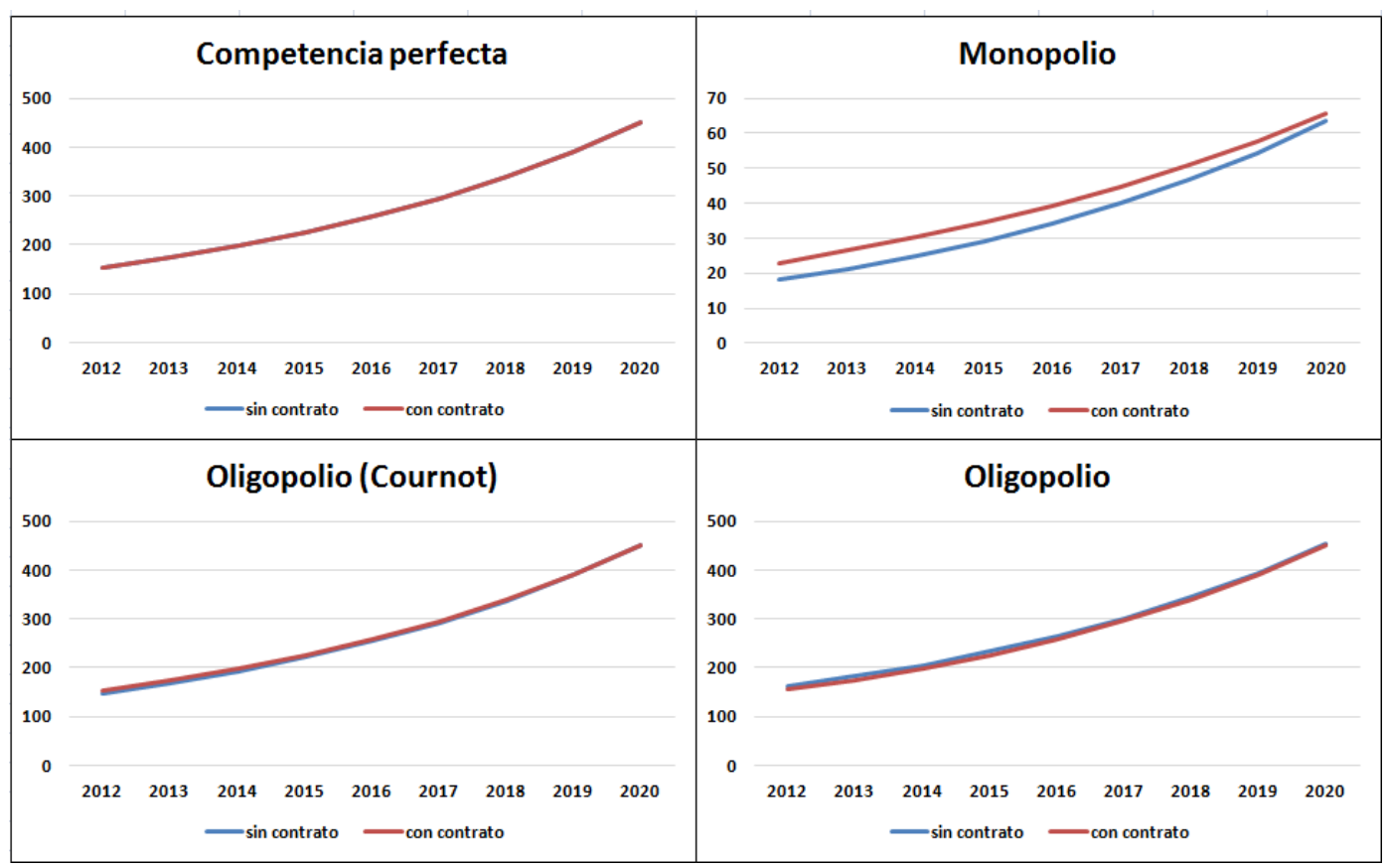

Fuente: Elaboración propia.

Con respecto a la capacidad de la tecnología hidráulica (gráfico 5) se observa una evolución creciente, producto de la fuerte inversión en nuevas centrales con nueva capacidad o en el incremento de la capacidad de las centrales ya operativas. El efecto de los contratos muestra un incremento de 5,09\% para el monopolio, 2,68\% para el oligopolio (Cournot), 1,56\% para el oligopolio con franja competitiva. Considerando el periodo que puedan operar las centrales hidráulicas a diferencia de las térmicas, se asume un incremento sustancial en sus inversiones. Se muestra que, bajo el escenario de monopolio, el generador tiene incentivo a incrementar su precio distorsionando el parque, disminuyendo su capacidad. Los contratos permitirían que tal disminución se suavice. También, se observa que el aumento de capacidad se muestra en los escenarios de competencia perfecta, que no cuenta con un efecto de los contratos; mientras que el oligopolio con franja competitiva, y el oligopolio (Cournot), muestran un efecto positivo.

La situación ante el uso de tecnologías térmicas muestra una leve reducción de la capacidad de generación en todos los casos hasta la mitad del periodo, seguido por una rápida recuperación, a excepción del monopolio (gráfico 6). El efecto de los contratos muestra un incremento de 0,75\% para el monopolio, 0,34\% para el oligopolio (Cournot), y una reducción del 0,59\% para el oligopolio con franja competitiva. Partiendo del caso de monopolio, que presenta una reducción de capacidad, se pueden considerar varios aspectos como el tiempo de operatividad de las centrales, el incremento de nuevas centrales con baja capacidad (diésel o residual), o la indisposición de éstas. En los escenarios restantes, la evolución de capacidad tiene el mismo patrón tendencial creciente. Si bien, dentro de las tecnologías térmicas no solo operan las de baja capacidad, sino las de ciclo simple y combinado (gas natural), el incremento de la inversión y la reducción de la capacidad hasta la mitad del periodo puede deberse en parte al retiro o conversión de las centrales operativas a diésel o residual a gas natural, lo que podría mantener inoperativas algunas centrales. Parte de la recuperación de la capacidad de generación haría suponer que es resultado de una reconfiguración óptima del parque generador. 
Gráfico 5. Capacidad de generación hidráulica (MW).

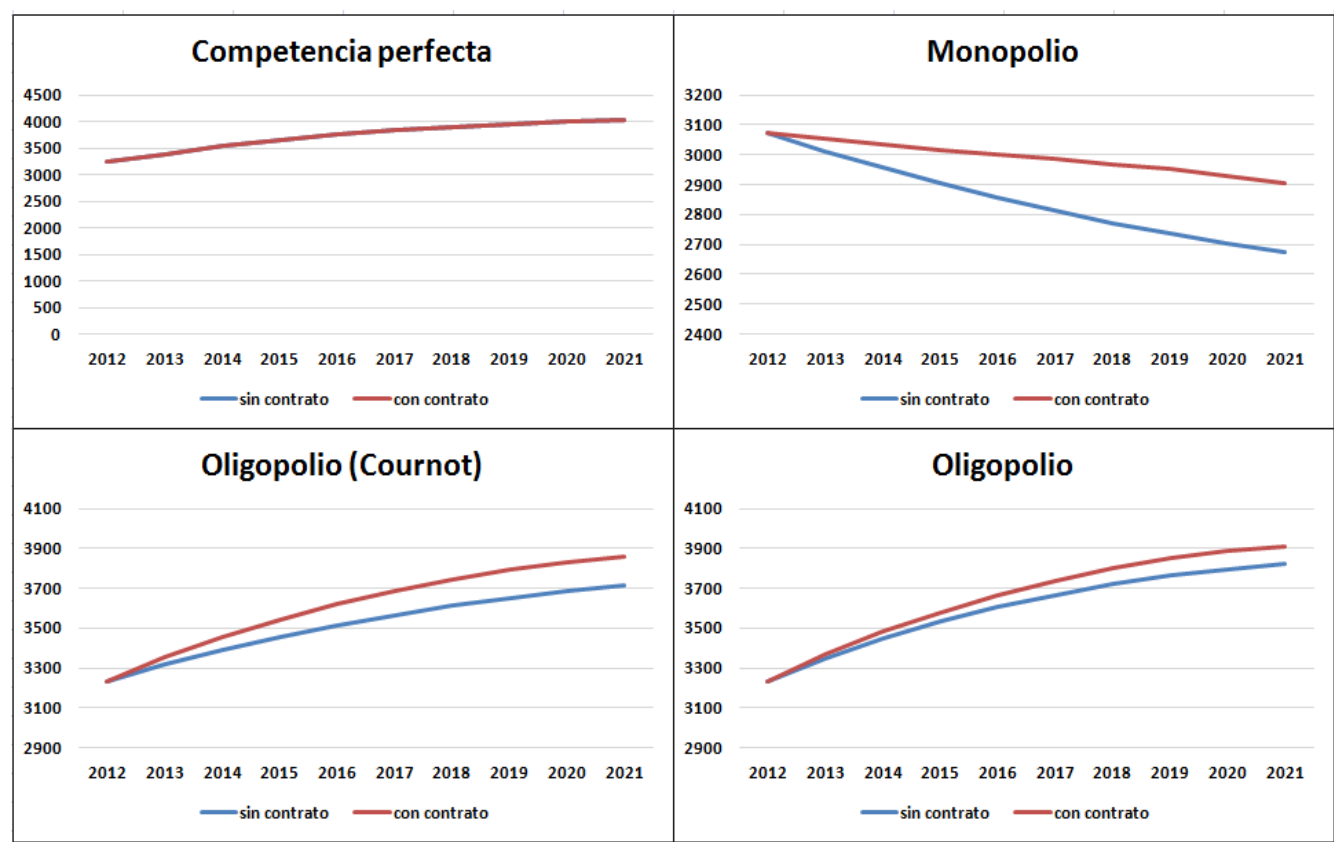

Fuente: Elaboración propia.

Gráfico 6. Capacidad de generación térmica (MW).

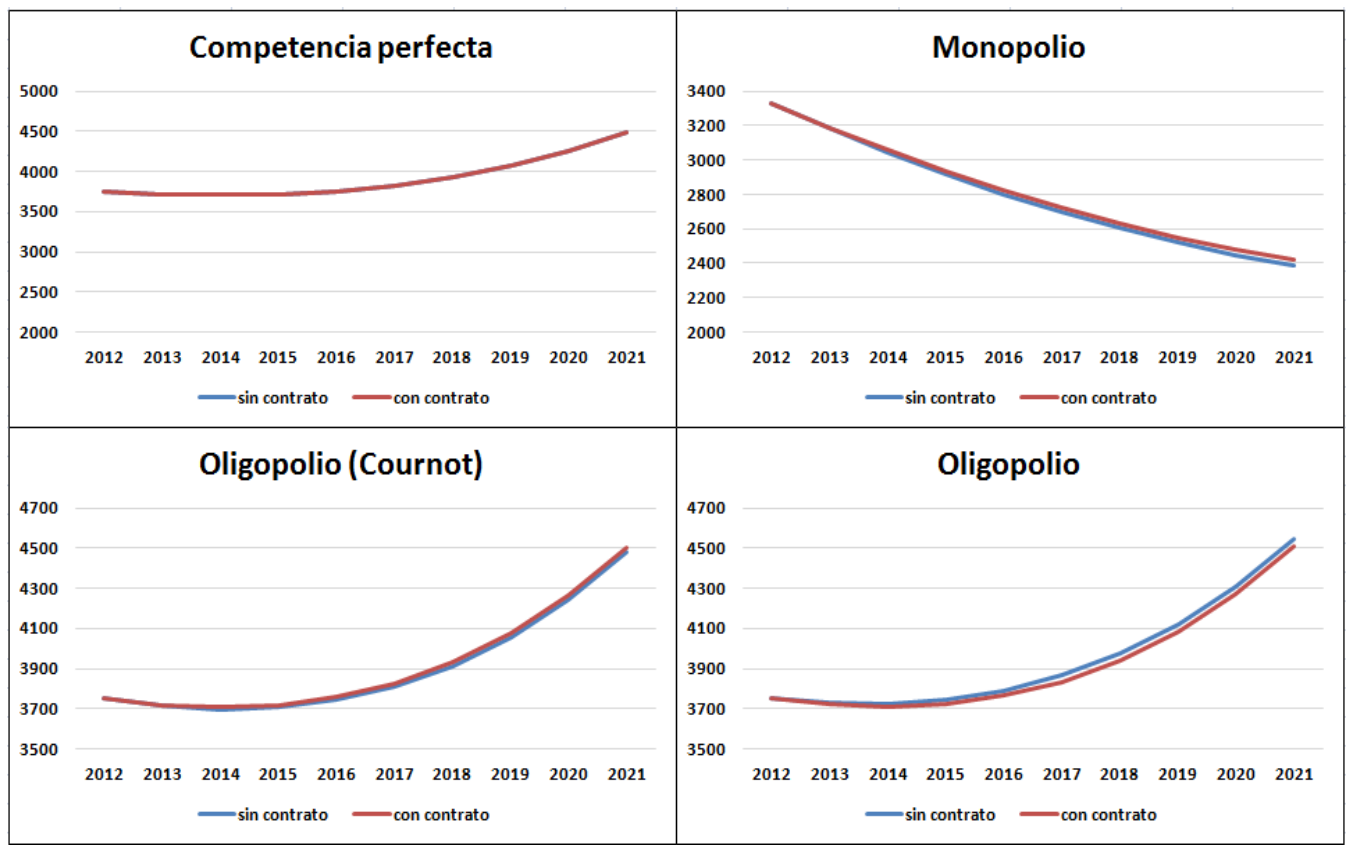

Fuente: Elaboración propia.

Los grupos económicos producen energía dependiendo de su inversión (capacidad), los competidores, el contrato determinado y el precio al que se venda por bloque. Dado esto, la cantidad producida bajo tecnología hidráulica muestra un componente creciente mayor a la térmica (gráfico 7). El efecto de los contratos muestra un incremento de $24,95 \%$ para el monopolio, 3,81\% para el oligopolio (Cournot), 3,67\% para el oligopolio con franja competitiva. Bajo el escenario de competencia perfecta, los grupos tienen incentivos por el incremento de la capacidad de generación en un contexto donde no existen indicios de poder de mercado ni grupos dominantes. En el escenario del monopolio, tiene incentivo a indisponer algunas centrales o 
declararlas inoperativas, por lo que se observa que a partir del cuarto año se produce una disminución de la producción, cuyo efecto es significativo con la incorporación de los contratos. Considerando la presencia de una franja competitiva dentro de un oligopolio, se muestra que la producción de energía es mayor que un escenario de oligopolio (Cournot).

Gráfico 7. Cantidad producida con tecnología hidráulica (MWh).

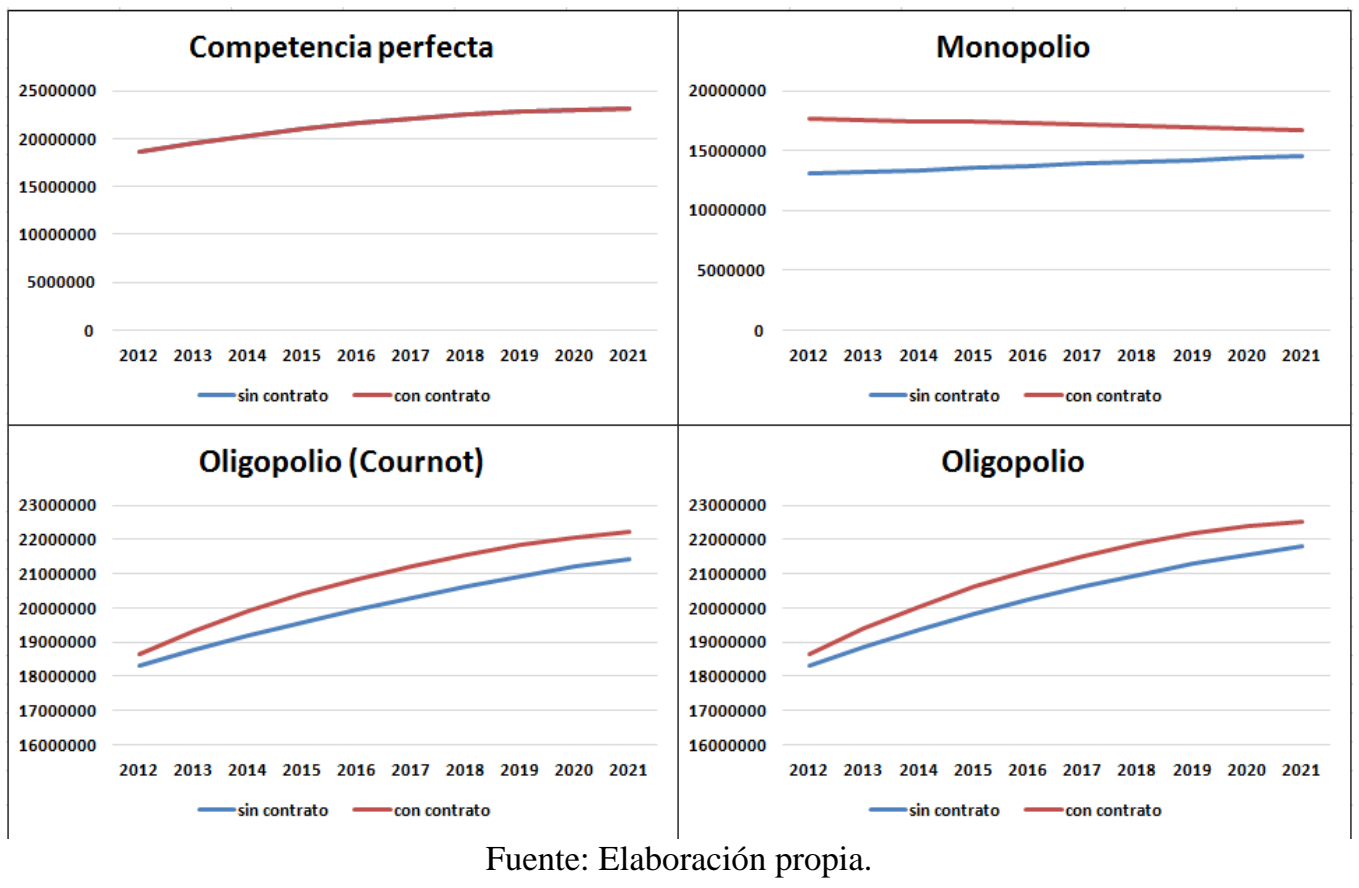

La producción de los grupos de electricidad bajo tecnología térmica, dada la configuración del parque generador y las necesidades en el horario fuera de punta, muestran un nivel menor a la hidráulica (gráfico 8). El efecto de los contratos muestra un incremento de 37,13\% para el monopolio, 8,48\% para el oligopolio (Cournot), 4,58\% para el oligopolio con franja competitiva. En el escenario de competencia perfecta la evolución de la producción no muestra cambios considerando contratos a largo plazo. Ante la presencia de un solo grupo generador, este cuenta con incentivo para dejar de ir produciendo, producto de una disminución de la capacidad de generación; mientras que, con los contratos, se observa que la situación mejora considerablemente manteniendo una tendencia constante. Los escenarios restantes incrementan la producción por la posible incorporación de nuevas tecnologías que puedan darse ante la reconversión de centrales y un paulatino crecimiento de la capacidad de generación.

Adicionalmente se muestra la composición del parque generador en los diferentes escenarios de mercado, bajo la incorporación de contratos (gráfico 9). La situación de competencia perfecta refleja un comportamiento creciente de capacidad de la tecnología hidráulica, lo que ha de suponer que los grupos con centrales térmicas tengan incentivos a convertirse en más eficientes con la entrada de gas natural, efecto que se muestra a partir del cuarto periodo de análisis. Caso contrario se observa cuando existe un solo grupo en el sector, con un fuerte desincentivo de inversión en tecnología térmica, así como en hidráulica. En este caso el grupo ejerce poder de mercado en menor medida a la situación sin contratos, aliviando la distorsión del parque. El efecto de los contratos es más notorio cuando los grupos compiten a lo Cournot o con la presencia de una franja competitiva, ya que habrá mayor incentivo de inversión con este último reflejado en una mayor capacidad de generación hidráulica; situación similar se muestra en la térmica, por lo que el parque generador bajo el escenario de oligopolio con franja competitiva resulta ser más eficiente. 
Gráfico 8. Cantidad producida con tecnología térmica (MWh).

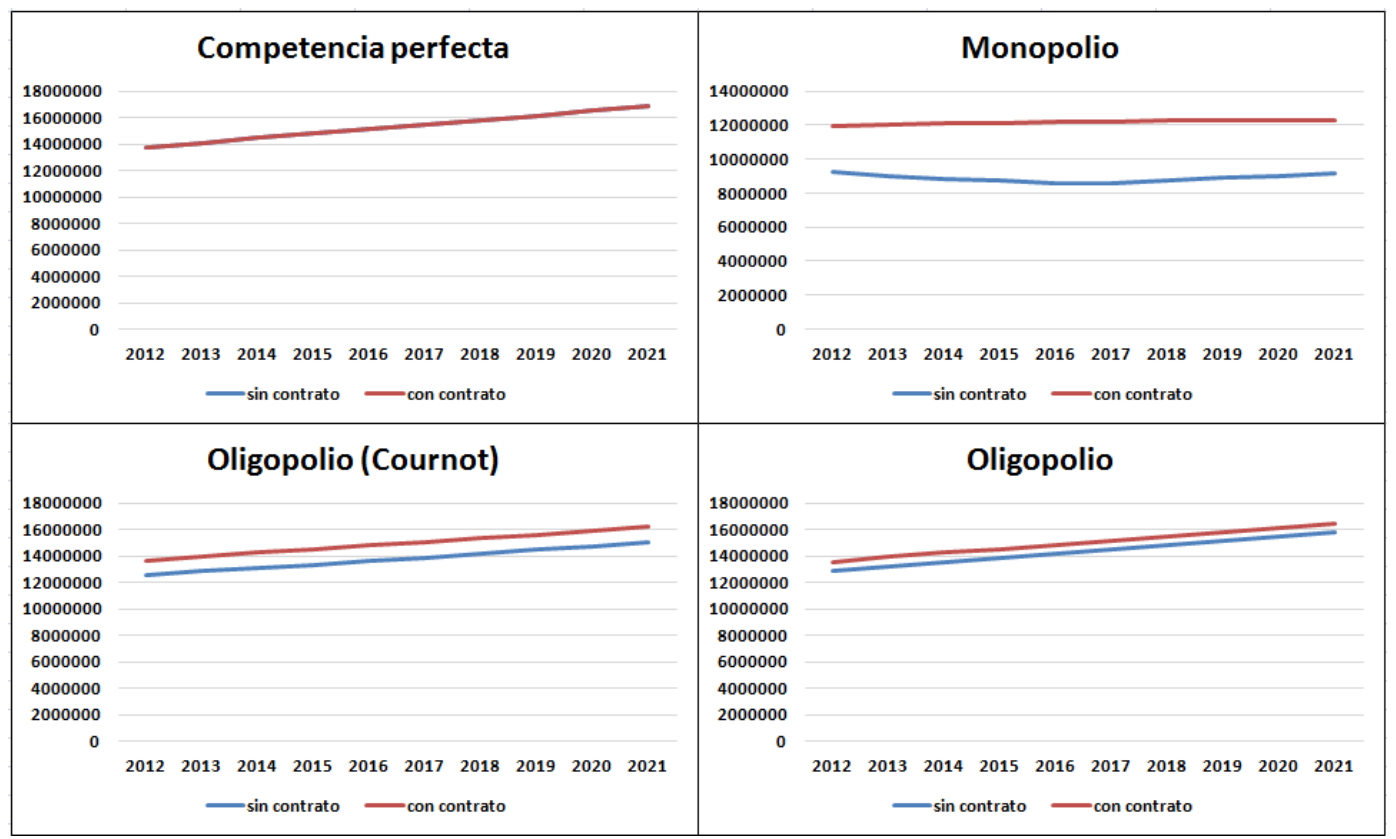

Fuente: Elaboración propia.

Gráfico 9. Composición del Parque Generador (\%).

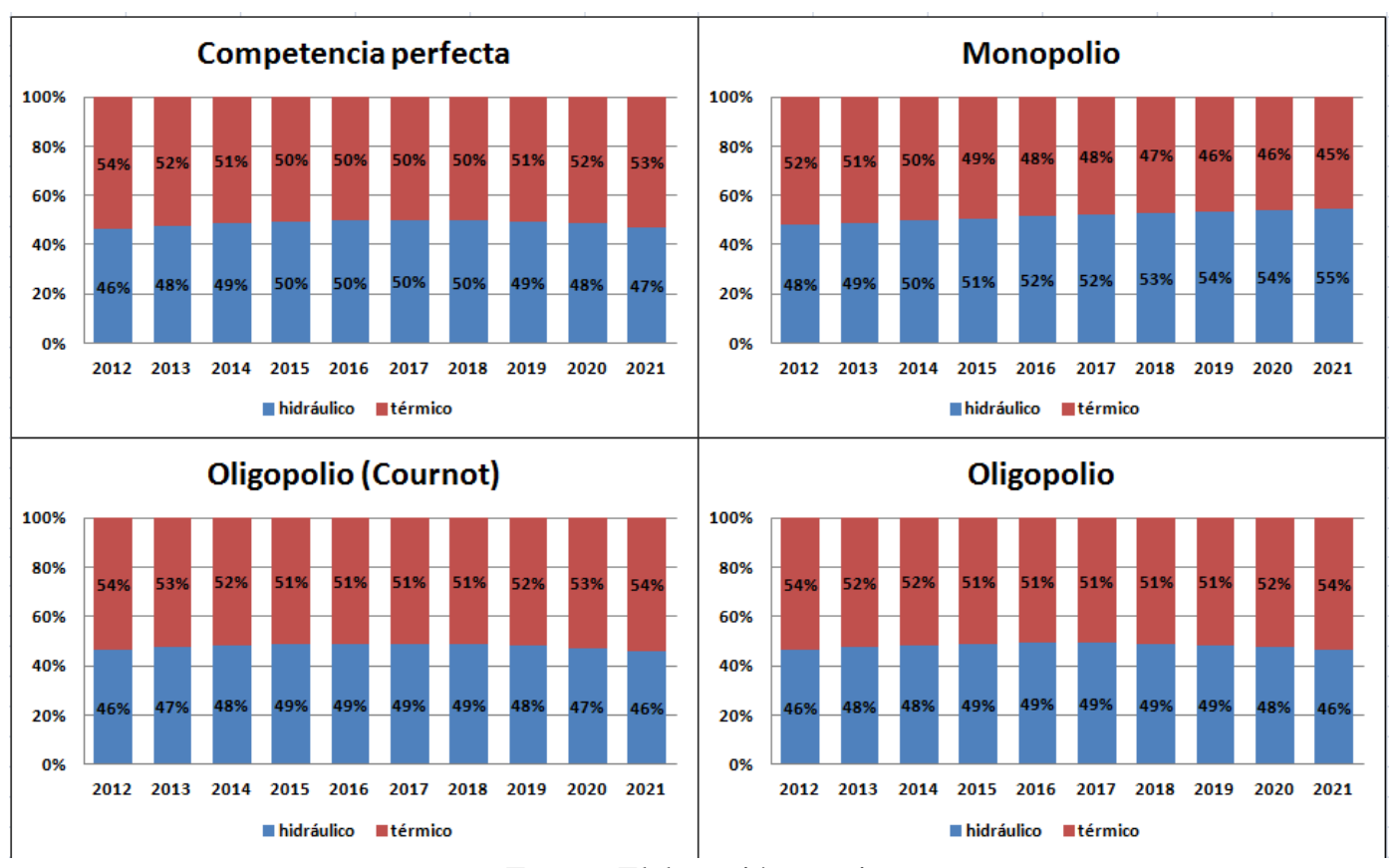

Fuente: Elaboración propia.

\section{Conclusiones y recomendaciones.}

En la presente investigación se realizó un análisis de la actividad de generación eléctrica bajo el supuesto de que el mercado peruano se encontraba liberalizado y se muestra el impacto de un esquema de contratos de largo plazo. Dentro de este contexto se hizo un análisis de un modelo de oligopolio con franja competitiva, dado el nivel de concentración de mercado, considerando otros escenarios, desde la presencia de un solo grupo que configura su decisión de manera independiente (monopolio) hasta la de grupos que son tomadores de precios (competencia 
perfecta), donde se muestra su influencia sobre las variables de decisión que actualmente se encuentran reguladas. Para esto se trató de ejemplificar dicho comportamiento de los grupos bajo un modelo que considera un enfoque de teoría de juegos.

Los resultados muestran que contratando el 70\% de energía hidráulica y el 66\% de energía térmica en el periodo base, la inversión en capacidad con tecnología hidráulica para el contexto de oligopolio con franja competitiva, para un horizonte temporal de 10 años, incrementa en 7,18\% (sobreinversión) y con tecnología térmica disminuye en 2,27\% (subinversión); mientras que la producción, con tecnología hidráulica incrementa en 3,67\%, mientras que bajo tecnología térmica, 4,58\%. El precio en hora base disminuye en $9,83 \%$ y en hora pico, 4,45\%. En ese sentido, se muestra que a pesar de que en los diferentes escenarios puedan existir indicios de poder de mercado, los contratos tienen un efecto positivo en inversión en capacidad y producción, y un efecto negativo sobre los precios. Los grupos económicos de generación tienen mayor incentivo a invertir en capacidad, incrementando la producción, dado que ya no podrían manipular el precio en el mercado spot, porque no tendría efecto sobre sus beneficios. Asimismo, considerando las tecnologías operativas, lo que se observa es que habría incentivos para sobreinvertir en capacidad en tecnología hidráulica y subinvertir en capacidad en tecnología térmica para el escenario que considera franja competitiva, por lo que la adecuación del parque no reflejaría indicios de distorsión.

Ante una posible entrada de nuevos grupos económicos en los escenarios analizados, se muestra mejores condiciones para el sector considerando un oligopolio con franja competitiva, lo que permitiría disminuir los precios por bloques de horario e incrementar la inversión mejorando la capacidad de generación.

De esta manera se incorporan una serie de recomendaciones que podrían ayudar a fomentar una generación eficiente reduciendo la exposición del parque generador a incrementos en el precio y posibles racionamientos, con efectos a terceros.

- Si bien es cierto que un mercado liberalizado no funcionaría de una forma completamente competitiva, se podría fomentar una disminución paulatina de la intervención administrativa, ya que los contratos de largo plazo tienen un efecto positivo sobre las variables de elección.

- Considerando que hay un grupo reducido de grupos económicos de generación eléctrica, el análisis por tipo de mercado responde parcialmente a proponer que a pesar que el costo fijo de inversión de las centrales hidroeléctricas es mayor que las térmicas, se debería fomentar este tipo de tecnología, ya que el efecto sobre el consumidor es menor y mejora la composición de parque generador.

- También es importante considerar un mecanismo de subastas anticipadas con un mayor plazo para promover el desarrollo del mercado e inversiones de los grupos participantes.

- Bajo la composición de la tecnología térmica que considera la entrada del gas natural se pueden promover iniciativas de reconversión gradual de centrales térmicas operativas a diésel y residual, fomentando mejoras en el parque generador y en las tarifas a los consumidores en el bloque de horario pico.

Sobre la base de las limitaciones descritas en la metodología, se puede considerar como extensión del modelo para futuros trabajos sobre el sector eléctrico, la incorporación de tecnologías renovables no convencionales en el parque generador, mediante algún tipo de modelamiento estocástico, dada la intermitencia de dichos recursos. Se pueden adoptar restricciones complementarias de transmisión eléctrica que afectan la configuración del parque generador, a fin de caracterizar problemas de congestión. Esto está ampliamente documentado a través de modelos de unit commitment. También, considerando la variabilidad de la demanda de 
electricidad, se podría realizar un análisis de series de tiempo para capturar un patrón de demanda, de tal manera que no se asuma una representación lineal, así como profundizar sobre la curva de carga. Asimismo, se podría replicar el modelo bajo condiciones actuales y representarlo bajo un enfoque de juegos cooperativos de tal manera que se pueda calcular un valor de Shapley como un equilibrio industrial.

Por otro lado, la estructura oligopólica puede extenderse a un análisis bajo el modelo KrepsSheinkman, que se asemejaría a lo que pasa en el despacho. También, se puede considerar la incertidumbre como factor de la decisión de inversión, dado que se omiten aspectos políticos que puedan darse y no se toma en cuenta el cauce hídrico para las hidroeléctricas, tampoco la volatilidad del precio de los combustibles usados por térmicas.

\section{Agradecimientos}

Este documento es una adaptación de la tesis de licenciatura en economía de Arnold Rivasplata Ramírez de la Pontificia Universidad Católica del Perú. Agradecemos los valiosos comentarios y sugerencias de los profesores Abelardo Jordán (PUCP - Departamento de Ciencias), Alfredo Dammert (PUCP - Maestría de Regulación de Servicios Públicos), Pierre-Olivier Pineau (HEC Montréal - Département de Sciences de la Décision) y Arturo Vásquez (GĚRENS - Escuela de Gestión, PUCP - Departamento de Economía). Los errores u omisiones son exclusivamente responsabilidad de los autores.

\section{Referencias}

Arellano, M.S., \& Serra, P. (2005). Market power in price-regulated power industries. Universidad de Chile. Centro de Economía Aplicada.

Burger, A., \& Ferstl, R. (2008). Generation capacity investment in oligopolistic electricity markets under uncertainty. Electricity Market. 5th International Conference on European.

Dammert, A., García C., \& Molinelli, F. (2010). Regulación y supervisión del sector eléctrico (1 ${ }^{a}$ ed.). Perú: Fondo Editorial PUCP.

Dammert, A., García C., Quiso, L. (2005). Dinámica de la Inversión en Generación de Electricidad en el Perú. XXII Encuentro de Economistas del Banco Central de Reserva del Perú. OSINERG.

De la Cruz, R., \& García, R. (2002). Mecanismos de competencia en generación de energía y su impacto en la eficiencia: el caso peruano. CIES.

Gallardo, J., García, R., \& Pérez-Reyes, R. (2005a). Determinantes de la inversión en el sector eléctrico peruano. OSINERG. Documento de trabajo 3.

Gallardo, J., García, R., \& Távara, J. (2005b). Instituciones y Diseño de Mercado en el Sector Eléctrico Peruano: Análisis de la Inversión en Generación. CIES.

Genc, T., Reynolds, S., \& Sen, S. (2007). Dynamic oligopolistic games under uncertainty: a stochastic programming approach. Journal of Economic Dynamics and Control, 31(1), 55-80.

Genc, T., \& Thille, H. (2011). Investment in Electricity Markets with Asymmetric Technologies. Energy Economics, 33(3), 379-387. 
González, J. (2008). Desarrollo de inversiones en mercados eléctricos oligopólicos. Tesis de Maestría. Pontificia Universidad Católica de Chile. Escuela de Ingeniería.

MINEM (2012). Subsector Eléctrico. Documento Promotor 2012. Recuperado de: http://www.minem.gob.pe/archivos/Documento_Promotor_2012.pdf

Nagurney, A. (2002). Oligopolies and Nash Equilibrium. University of Massachusetts. Isenberg School of Management. Recuperado de: https://pdfs.semanticscholar.org/f4dc/555273aa082542c9ca5e021f2ceffef416ee.pdf

Osinergmin (2011). Operación del Sector Eléctrico. Boletín Anual 2011. http://www2.osinerg.gob.pe/Publicaciones/pdf/BolAnualSectorElectric/Boletin-AnualSEIN_2011.pdf

Pineau, P., \& Murto, P. (2003). An oligopolistic Investment Model of Finnish Electricity Market. Annals of Operations Research, 121(1), 123-148.

Pineau, P., Rasata, H., \& Zaccour, G. (2011). A dynamic oligopolistic electricity market model with interdependent segments. The Energy Journal, 32(4), 183-217.

Rasata, H., \& Zaccour, G. (2010). An empirical investigation of open-loop and closed-loop equilibrium investment strategies in an electricity oligopoly market. Les Cahiers du GERAD 54.

Zoettl, G. (2008). Investment decisions in liberalized electricity markets: A framework of peak load pricing with strategic firms. University of Cologne. Working paper series in economics 38. 
ANEXO: REPRESENTACIÓN EXTENSA DEL MODELO

$$
\begin{gathered}
L_{j}^{t}=\bar{Q}_{c j} \cdot \bar{p}_{c j}+p^{h p}\left(Q_{t}^{h p}\right)\left[Q_{j}-Q_{c j}\right]_{t}^{h p}+p^{h f p}\left(Q_{t}^{h f p}\right)\left[Q_{j}-Q_{c j}\right]_{t}^{h f p}-C^{H}\left(q^{H, h p}+q^{H, h f p}, K_{t}^{H}\right) \\
-C^{T}\left(q^{T, h p}+q^{T, h f p}, K_{t}^{T}\right)-\Psi^{H}\left(I_{t}^{H}\right)-\Psi^{T}\left(I_{t}^{T}\right)+\sum_{i \in\{H, T\}} \lambda_{t+1}^{i}\left(I_{t}^{i}-\delta K_{t}^{i}\right)+\sum_{i \in\{H, T\}} \xi_{t}^{i}\left(h . K_{t}^{i}-q_{t}^{i, h}\right)
\end{gathered}
$$

Resolviendo las condiciones de primer orden:

$$
\begin{gathered}
\frac{\partial L_{j}^{t}}{\partial q_{j}^{h f p}} \leq 0 ; \quad q_{j}^{h f p} \geq 0 ; \quad q_{j}^{h f p} \frac{\partial L_{j}^{t}}{\partial q_{j}^{h f p}}=0 \\
\frac{\partial L_{j}^{t}}{\partial q_{j}^{h p}} \leq 0 ; \quad q_{j}^{h p} \geq 0 ; \quad q_{j}^{h p} \frac{\partial L_{j}^{t}}{\partial q_{j}^{h p}}=0 \\
\frac{\partial L_{j}^{t}}{\partial I_{j}^{h f p=H}} \leq 0 ; \quad I_{j}^{h f p=H} \geq 0 ; \quad I_{j}^{h f p=H} \frac{\partial L_{j}^{t}}{\partial I_{j}^{h f p=H}}=0 \\
\frac{\partial L_{j}^{t}}{\partial I_{j}^{h p=T}} \leq 0 ; \quad I_{j}^{h p=T} \geq 0 ; \quad I_{j}^{h p=T} \frac{\partial L_{j}^{t}}{\partial I_{j}^{h p=T}}=0 \\
\frac{\partial L_{j}^{t}}{\partial \xi_{j}^{h f p=H}} \geq 0 ; \quad \xi_{t}^{H} \geq 0 \quad ; \quad \xi_{t}^{H}\left(h f p K_{t}^{H}-q_{t}^{H, h f p}\right)=0 \\
\frac{\partial L_{j}^{t}}{\partial \xi_{j}^{h p=T}} \geq 0 ; \quad \xi_{t}^{H} \geq 0 \quad ; \quad \xi_{t}^{T}\left(h p K_{t}^{T}-q_{t}^{T, h p}\right)=0
\end{gathered}
$$

Representación de variables de coestado:

$$
\begin{gathered}
\frac{\partial L_{j}^{t}}{\partial K_{t}^{H}}=0 \rightarrow-\kappa I_{t}-\delta \lambda_{t+1}^{H}+\xi_{t}^{H} h f p=0 \\
\lambda_{t+1}^{H}-\frac{\lambda_{t}^{H}}{\beta}=\kappa I_{t}+\delta \lambda_{t+1}^{H}-\xi_{t}^{H} h f p \\
\frac{\partial L_{j}^{t}}{\partial K_{t}^{T}}=0 \rightarrow \phi \frac{\gamma}{\phi+1}\left(\frac{q_{t}^{T}}{K_{t}^{T}}\right)^{\phi+1}-\delta \lambda_{t+1}^{T}+\xi_{t}^{T} h p=0 \\
\lambda_{t+1}^{T}-\frac{\lambda_{t}^{T}}{\beta}=-\phi \frac{\gamma}{\phi+1}\left(\frac{q_{t}^{T}}{K_{t}^{T}}\right)^{\phi+1}+\delta \lambda_{t+1}^{T}-\xi_{t}^{T} h p
\end{gathered}
$$

Representación de las ecuaciones de estado:

$$
\begin{aligned}
& K_{t+1}^{H}=(1-\delta) K_{t}^{H}+I_{t}^{H} \\
& K_{t+1}^{\mathrm{T}}=(1-\delta) K_{t}^{T}+I_{t}^{T}
\end{aligned}
$$

